\title{
28 Research Square \\ Generation of functional heart organoids from mouse embryonic stem cells.
}

\section{Jiyoung Lee ( $\nabla$ jlee.epgn@mri.tmd.ac.jp )}

Department of Epigenetics, Medical Research Institute, Tokyo Medical and Dental University https://orcid.org/0000-0001-7717-8104

\section{Fumitoshi Ishino ( $\square$ fishino.epgn@mri.tmd.ac.jp )}

Department of Epigenetics, Medical Research Institute, Tokyo Medical and Dental University https://orcid.org/0000-0001-8458-6069

\section{Akito Sutani}

Department of Epigenetics, Medical Research Institute, Department of Pediatrics and Developmental Biology, Tokyo Medical and Dental University

\section{Rin Kaneko}

Department of Epigenetics, Medical Research Institute, Tokyo Medical and Dental University

\section{Method Article}

Keywords: Mouse, heart development, organoids, extracellular matrix, embryonic stem cells

Posted Date: September 3rd, 2020

DOl: https://doi.org/10.21203/rs.3.pex-1078/v1

License: (c) (1) This work is licensed under a Creative Commons Attribution 4.0 International License.

Read Full License 


\section{Abstract}

For the understanding of the spatiotemporal regulation of cardiogenesis, it is important to generate in vitro model of hearts. This protocol introduces how to generate heart organoids from mouse embryonic stem cell-derived embryoid bodies (EB) in the presence of the laminin-entactin (LN/ET) complex and fibroblast growth factor 4 (FGF4) by three consecutive steps, 1) the culture of ESCs, 2) in vitro differentiation of ESCs into EBs and 3) in vitro culture of EBs for the generation of heart organoids. The generated heart organoids possess structural and functional capacity similar to atrial and ventricular parts of in vivo embryonic heart. This simplified protocol also provides a promising research tool with a broad range of applications, including drug testing.

\section{Introduction}

Mammalian heart development involves differentiation of diverse cell types, including atrial and ventricular muscle cells known as cardiomyocytes (CMs), specific myocytes constituting conducting tissue such as Purkinje fibers, smooth muscle (SM) cells, and non-muscle cells including endothelial cells (ECs) and neuronal cells. Many researchers have developed an in vitro system that mimics in vivo cardiogenesis in order to better understand the function of heart, and provide technical advantages for drug testing and model for cardiac pathology. However, three-dimensional (3D) heart organoidgeneration itself has been hampered by the structural complexity of the heart.

For 3D culture methods using embryonic stem cells (ESCs) or adult stem cells, ECMs including Matrigel and laminin were widely used for in vitroorganogenesis, including the development of the brain ${ }^{1}$, stomach $^{2}$, kidney ${ }^{3}$, and intestinal tissues ${ }^{4}$. In case of 3D heart modeling, FGF can be thought as a good candidate given that FGF and FGFR1 are required for cardiac gene expression.

This heart organoid model system using the laminin-entactin (LN/ET) complex ${ }^{5}$ in the ECM and FGF4 ${ }^{6}$ generates heart organoids with atrium- and ventricle-like structures containing $\mathrm{CM}$, conducting tissues, SM and ECs that exhibited myocardial contraction and action potentials. Also, these heart organoids exhibited the ultrastructural, histochemical and gene expression profile similarities to embryonic hearts via consecutive morphological changes like self-organization and, therefore, without the requirement of complex differentiation protocols.

Theses heart organoids can be used to study mouse heart development and can also provide an effective tool with applications, such as drug safety testing for hearts.

\section{Reagents}

口Gelatin (Nacalai Tesque, 16631-05)

[DMEM medium (Sigma, D5796) 
[PBS (-) (Wako, 166-23555)

पKnockout DMEM (Gibco, 10829-018)

\100 × NEAA (Wako, 139-15651)

[EmbryoMax $100 \times$ Nucleoside for ES cells (Millipore, ES-008-D)

QPenicillin-streptomycin/glutamine (Sigma-Aldrich, G6784)

[CHIR99021 (GSK-3ßinhibitor; Axon, 1386)

GPD0325901 (MAPK inhibitor; Wako, 162-25951)

[ESGRO Mouse LIF medium Supplement (LIF) (Millipore, ESG1107)

[Trypsin-EDTA solution (Sigma, T4049)

[Fetal Bovine Serum (FBS) (Gibco, 10437-028)

口Laminin/Entactin Complex (BD, 354259)

LDMEM/F12 medium (Gibco, 11330-032)

[Penicillin/Streptomycin (Sigma, P4333)

पKnockout Serum Replacement (KSR) (Gibco, 10828-028)

ZiMatrix-411 (Nippi, 892 042)

๑Sodium pyruvate (Sigma, S8636)

पß-Mercaptoethanol (Gibco, 21985)

GL-glutamine (Sigma, G7513)

पProgesterone (Sigma, P6149)

$\square \beta$-estradiol (Sigma, E2257)

[Insulin (Sigma, 16634)

पTransferrin (Sigma, T8158)

पSodium selenite (Sigma, S9133)

पRecombinant human FGF4 (R\&D systems, 235-F4) 
口Recombinant human BMP4 (R\&D systems, 314-BP-010)

पBIO (Cayman chemical, 13123)

\section{Reagent setup}

IMEF medium: 10\% FBS, 2mM L-glutamine, penicillin/Streptomycin (1/100 dilution) in DMEM medium GES medium: $20 \% \mathrm{KSR}, 100 \mu \mathrm{M} \beta$-mercaptoethanol, $1 \times$ NEAA, $1 \times$ EmbryoMax Nucleoside, penicillinstreptomycin/glutamine (1/100 dilution) in Knockout DMEM

पFBS medium: 20\% FBS, $100 \mu \mathrm{M}$ ß-mercaptoethanol, $1 \times$ NEAA, $1 \times$ EmbryoMax Nucleoside, penicillinstreptomycin/glutamine (1/100 dilution) in Knockout DMEM

पHeart organoid medium: 20\% KSR, 1 mM sodium pyruvate, $100 \mu \mathrm{M} \beta$-mercaptoethanol, $2 \mathrm{mM} \mathrm{L-}$ glutamine, penicillin/streptomycin (1/100 dilution), $60 \mathrm{ng} / \mathrm{ml}$ progesterone, $30 \mathrm{ng} / \mathrm{ml} \beta$-estradiol, $5 \mu \mathrm{g} / \mathrm{ml}$ insulin, $20 \mu \mathrm{g} / \mathrm{ml}$ transferrin, and $30 \mathrm{nM}$ selenite in DMEM/F12 medium.

\section{Equipment}

uBio clean bench (Sanyo, MCV-B91F)

口Biological safety cabinet (Sanyo, MHE-130AJ)

aNitrile, power free exam gloves (Kimberly-Clark, KC100)

$\square$ Cell culture humidified $\mathrm{CO} 2$ incubator $37^{\circ} \mathrm{C}, 5 \% \mathrm{CO} 2$

(Sanyo, MCO-19A1C (UV), MCO-19A1C)

[Water bath set at $37^{\circ} \mathrm{C}$ (TAITEC, Thermo minder SD mini)

QPhase contrast microscope (Olympus, CKX41)

口Table top centrifuge (TOMY, Low speed centrifuge LCX-100)

aMicrocentrifuge, cooling (TOMY, MX-150)

ه12-well cell culture plates (IWAKI, 3815-012)

『6-well cell culture plates (IWAKI, 3810-006)

पPrime surface 96-well U U-bottom 96-well culture plates (Sumiron, MS-9096U)

\8-well chamber slides (Falcon, 35418) 
ه15 ml Centrifuge Tubes, sterile (STAR, RSV-15CT2)

ه50 ml Centrifuge Tubes, sterile (STAR, RSV-50CT2)

$₫ 1.5 \mathrm{ml}$ microtubes (Axygen, MCT-175-C)

aDisposable serological $2 \mathrm{ml}$ pipettes, sterile (True line, TR3006)

aDisposable serological $5 \mathrm{ml}$ pipettes, sterile (True line, TR3007)

पDisposable serological $10 \mathrm{ml}$ pipettes, sterile (True line, TR3008)

aDisposable serological $25 \mathrm{ml}$ pipettes, sterile (True line, TR3009)

\8-channel pipettor (PipetPAL)

$\square$ Sterile reagent reservoirs (Integra, \#4312)

[Wide bore $200 \mathrm{ml}$ pipette tips (RAININ, BioClean Ultra, RT-UNV-A-200 ml)

aDissection forceps (FST by DUMONT, \#5 INOX)

[Stereomicroscope (Leica, MZ16F)

\section{Procedure}

\section{Culture of ESCs}

1. Coat 12 -well plates with $1 \mathrm{ml}$ of $0.2 \%$ gelatin solution per well and place at room temperature (RT) for over 30 minutes.

2. Remove $0.2 \%$ gelatin solution by aspirating.

3. Seed mitomycin treated mouse embryonic fibroblast (MEF) feeder cells $(100,000$ cells per well, 12 -well plate) in $1 \mathrm{ml}$ of MEF medium and incubate at $37^{\circ} \mathrm{C}, 5 \% \mathrm{CO} 2$ incubator for 1 day.

4. Wash MEF plates with $1 \mathrm{ml}$ of PBS (-) and remove PBS (-).

5. Seed murine ESCs $(100,000$ cells per well, 12 -well plate) on MEF plates in $1 \mathrm{ml}$ of KSR based ESC medium (ES medium) supplemented with CHIR99021 (final 3mM), PD0325901 (final 1mM), and LIF (1000 Unit/ml) and incubate at $37^{\circ} \mathrm{C}, 5 \% \mathrm{CO} 2$ incubator.

6. Change ES medium every day.

7. After 2 days, aspirate culture medium and wash the cells with $1 \mathrm{ml}$ of PBS (-).

8. Add $0.4 \mathrm{ml}$ of $0.25 \%$ Trypsin-EDTA solution and incubate at $37^{\circ} \mathrm{C}$ for 3 minutes. 
9. Add $1 \mathrm{ml}$ of $10 \%$ FBS-DMEM medium and pipette cells with $1000 \mathrm{ml}$ tip.

10. Count the number of cells by using hemacytometer.

11. Pool the cells into a $15 \mathrm{ml}$ tube and centrifuge at $1,500 \mathrm{rpm}$ for 5 minutes.

12. Aspirate medium and resuspend the cells with ES medium supplemented with CHIR99021 (final 3mM), PD0325901(final 1mM), and LIF (1000 Unit/ml).

13. Seed murine ESCs $\left(100,000\right.$ cells per well, 12 -well plate) on MEF plates and incubate at $37^{\circ} \mathrm{C}, 5 \% \mathrm{CO} 2$ incubator.

\section{EB formation}

1. Prepare 6-well culture plates coated with $0.2 \%$ gelatin solution, before trypsinization of ESCs.

2. Aspirate culture medium from 2 days cultured ESCs and wash the cells with $1 \mathrm{ml}$ of PBS (-).

3. Add $0.2 \mathrm{ml}$ of $0.25 \%$ Trypsin-EDTA solution and incubate at $37^{\circ} \mathrm{C}$ for $2-3$ minutes.

4. Add $1 \mathrm{ml}$ of $10 \%$ FBS-DMEM medium and pipette cells with $1000 \mathrm{ml}$ tip.

5. Pool the cells and transfer the cells into 6 -well plates coated with $0.2 \%$ gelatin and incubated at $37^{\circ} \mathrm{C}$ for 45 minutes to remove feeder cells.

6. Collect floating ESCs gently without contamination of feeder cells and count the number of cells by using hemacytometer.

7. Centrifuge at $1,000 \mathrm{rpm}$ for 5 minutes.

8. Aspirate medium and resuspend the cells with warm FBS-supplemented ES medium without LIF (FBS ES medium).

9. Seed $1,000-5,000$ ESCs in $200 \mathrm{ml}$ of FBS ES medium onto each well of a U-bottomed 96 -well plate by using 8-channel pipettor.

10. Incubate at $37^{\circ} \mathrm{C}, 5 \% \mathrm{CO} 2$ incubator for 4 days without changing medium. The cells form aggregate spheres on the next day of seeding.

\section{Generation of heart organoids}

Plate coating with LN/ET gel $\left(\underline{85.7} \mu \mathrm{gg} / \mathrm{cm}^{2}\right)$. 
1. Before coating plates, thaw $2 \mathrm{mg} / \mathrm{ml}$ of LN/ET complex on ice.

2. Add $30 \mathrm{ml}$ of LN/ET complex into a well of 8-well chamber slides.

3. Incubate 8-well chamber slides at RT for 2 hours.

In vitro culture of EBs for the generation of heart organoids

6. Warm the heart organoid medium and FGF4.

7. Mix the heart organoid medium with FGF4 $(30-60 \mathrm{ng} / \mathrm{ml})$.

8. Add $200 \mathrm{ml}$ of the medium in each well of LN/ET gel coated 8-well chamber slides.

9. Carefully transfer an EB (showing sphere morphology) cultured for 4 days by using $200 \mathrm{ml}$ tips, into a well of LN/ET gel coated 8-well chamber slides with the medium.

10. Maintain cultures in $5 \% \mathrm{CO} 2$ incubator at $37^{\circ} \mathrm{C}$.

11. Replace medium at day3, 5, 7, 9, 11,13,14, and 15.

12. From day9, use FGF4-heart organoid medium additionally supplemented BMP4 ( $50 \mathrm{ng} / \mathrm{ml})$, BIO (2.5 $\mu \mathrm{M})$, and LIF (1,000 units $/ \mathrm{ml})$. In case of long term culture of heart organoids, such as over 15 days culture, carefully transfer heart organoids into iMatrix-411-coated $\left(10.7 \mu \mathrm{g} / \mathrm{cm}^{2}\right)$ chamber slides in $200 \mu \mathrm{l}$ of heart organoid medium.

13. Observe the morphogenesis of heart organoids under microscope. These heart organoids usually show the initial beating movement by 3 days, and continue to grow with morphogenetic changes.

14. Usually collect heart organoids over 9 days of culture after chamber formation. When transfer heart organoids for collecting or performing further analysis, use wide bore tip and/or fine dissection forceps under a stereomicroscope.

\section{Troubleshooting}

\section{Time Taken}

Day -6 : ES cell seeding $(0.5 \mathrm{~h})$

Day -5 : Medium change $(0.5 \mathrm{~h})$

Day -4 : EB formation (1.5 h) 
Day 0 : chamber slide coating with LN/ET complex ( $>2 \mathrm{~h}$; depends on the time of thawing LN/ET complex), preparation medium $(0.5 \mathrm{~h})$, transfer EBs $(0.5 \mathrm{~h})$

Day 1 to 15 : medium change $(0.5 \mathrm{~h})$

\section{Anticipated Results}

This protocol can generate structural and functional heart organoids.

\section{References}

1. Lancaster, M. A.et al. Cerebral organoids model human brain development and microcephaly. Nature 501, 373-379 (2013).

2. Noguchi, T. K.et al. Generation of stomach tissue from mouse embryonic stem cells. Nat. Cell Biol. 17, 984-993 (2015).

3. Taguchi, A. \& Nishinakamura, R. Higher-order kidney organogenesis from pluripotent stem cells. Cell Stem Cell 21, 730-746, e736 (2017).

4. Huch, M. \& Koo, B. -K. Modeling mouse and human development using organoid cultures. Development $142,3113-3125$ (2015).

5. Kleinman, H. K., Philp, D. \& Hoffman, M. P. Role of the extracellular matrix in morphogenesis. Curr. Opin.

Biotechnol. 14, 526-532 (2003).

6. Powers, C. J., McLeskey, S. W. \& Wellstein, A. Fibroblast growth factors, their receptors and signaling. Endocr. Relat. Cancer 7, 165-197 (2000).

\section{Acknowledgements}

This work was supported by the TMDU president grant. 